\title{
Conservative Treatment of Hemorrhoids: Results of an Observational Multicenter Study
}

\author{
Evgeny A. Zagriadskiǐ • Alexey M. Bogomazov • Evgeny B. Golovko
}

Received: June 20, 2018 / Published online: October 1, 2018

(C) The Author(s) 2018, corrected publication October/2018 hemorrhoids. All were prescribed MPFF-based conservative treatment. The effect of treatment on HD clinical signs and symptoms was assessed at two follow-up visits performed 5-7 days and 25-30 days after enrollment. Surgical and minimally invasive treatment could be performed from day 7 onwards if required.

Results: A total of 1952 patients were enrolled. Over the entire period of observation, MPFFbased conservative treatment was effective in 1489 (76.3\%) patients in eliminating the main clinical manifestations of disease, i.e., bleeding and prolapse of internal nodes. Invasive treatment was performed in $68(3.5 \%)$ patients with grade IV hemorrhoids and was combined with MPFF conservative treatment in 395 (20.2\%) patients with grades I-III hemorrhoids.

Conclusion: Conservative therapy with MPFF was beneficial for relieving hemorrhoidal symptoms in the majority of patients. MPFFbased treatment was most effective in patients with grade I and II hemorrhoids before irreversible degenerative changes in ligaments of the hemorrhoidal plexuses have occurred. It was also beneficial in preventing disease relapse in patients with more advanced HD and for promoting optimal conditions in the postoperative period.

Funding: Servier.

Keywords: Anal pain; Bleeding; Constipation; Hemorrhoidal disease; Micronized purified flavonoid fraction (MPFF)
E. B. Golovko

Clinic 'MEDSI MSK 12', Moscow, Russia 


\section{INTRODUCTION}

Hemorrhoids or hemorrhoidal disease (HD) is one of the most common diseases of the anorectal region. An epidemiologic study by Johanson and Sonnenberg in 1990 described the self-reported incidence of hemorrhoids in the USA of 10 million per year, corresponding to $4.4 \%$ of the population [1]. Prevalence studies suggest a rate of around 40\% [2]. Pregnancy is associated with an increased risk for hemorrhoids and there is a slightly increased prevalence in women compared with men $[3,4]$. In economically developed countries, HD takes a leading place among proctologic diseases [1]. Hemorrhoids become more common with age in both genders, with a peak incidence between the ages of 45 and 65 years [5]. HD represents a major medical and socioeconomic problem affecting substantial numbers of working-age individuals. Conservative treatment of hemorrhoids plays an essential role in preparing patients for either minimally invasive or surgical treatment $[6,7]$.

The Russian arm of the international CHORUS survey (Chronic venous and HemORrhoidal diseases evalUation for improvement of Scientific knowledge) was conducted to collect data on the clinical characteristics of patients presenting to physicians with symptomatic HD, and to obtain data on the effect of phlebotropic therapy on the evolution of clinical manifestations in patients with different degrees of internal hemorrhoids. The venotonic agent micronized purified flavonoid fraction (MPFF; Detralex, Servier, Russia) was selected as the phlebotropic treatment in this study because of its widely studied and proven efficacy on the main symptoms of HD in a large number of patients $[8,9]$.

\section{METHODS}

\section{Study Methodology}

This multicenter, observational study was conducted in nine ambulatory medical centers in different regions of Russia and enrolled 80 coloproctologists. The study period lasted
6 months (the first patient was enrolled at the end of October 2015 and the last in April 2016). The study was conducted in accordance with the principles of the Declaration of Helsinki (version adopted in Fortaleza, Brazil, in 2013) [10]. All patients provided written informed consent to participate in the study. The study protocol (No. DIM-05682-003-RUS) was approved by local institutional ethics committees.

Patients were examined for clinical signs and symptoms of HD according to current practice at their primary visit (V0) and at the follow-up visits which took place at 5-7 days (V1) and 25-30 days (V2). Information from the clinical examination, as well as assessment of treatment efficacy, was recorded in each patient's case report form (CRF). The following clinical manifestations of HD were recorded at each visit: anal pain, bleeding, swelling of nodes in the anal region, degree of prolapse of internal hemorrhoids, as well as anal itching and discomfort.

\section{Subjects}

The study included men and women over 18 years old who had complaints related to HD. The exclusion criteria were as follows: the presence of concomitant, severe systemic disorders; anal fissure; inflammatory bowel disease; colorectal cancer; or patient's inability to understand the nature of the study and follow the doctor's recommendations. In patients in whom the diagnosis of HD was verified on the basis of a clinical examination, conservative medical treatment including systemic phlebotropic therapy with MPFF was prescribed. The complex therapy of hemorrhoids included increasing dietary fiber (medications based on Plantago ovata seed shells, i.e., Mucofalk, Dr. Falk Pharma GmbH, Freiburg, Germany; Phytomucil norm, Probiotics International Ltd., UK), topical anti-inflammatory medications (suppositories and ointments), and non-steroidal anti-inflammatory drugs (NSAIDs), if indicated. The lack of effect of conservative treatment was an indication for more invasive treatment including surgery. The timing of 
invasive treatment was specified (minimally invasive and surgical treatment) in the study protocol. The nature of the invasive treatment carried out by physicians reflected national recommendations for the treatment of hemorrhoidal disease and did not differ from those accepted in worldwide clinical practice.

\section{Assessment of Conservative Treatment Efficacy}

The efficacy of conservative treatment was assessed at visits V1 (5-7 days) and V2 (25-30 days). Clinical signs of the disease, such as prolapse, bleeding, swelling, and pain after defecation were self-assessed by the patient using a yes/no questionnaire and a visual analogue scale (VAS) with ratings from 0 (no

Table 1 Baseline characteristics of patients included in the study

\begin{tabular}{|c|c|c|c|c|c|}
\hline Parameter & $\begin{array}{l}\text { Conservative } \\
\text { treatment } \\
(N=1489)\end{array}$ & $\begin{array}{l}\text { Minimally } \\
\text { invasive } \\
(N=396)\end{array}$ & $\begin{array}{l}\text { Invasive } \\
(N=67)\end{array}$ & $\begin{array}{l}\text { Total } \\
(N=1952)\end{array}$ & $\begin{array}{l}\text { Chi-square tests } \\
\text { asymp. Sig (2-sided) }\end{array}$ \\
\hline \multicolumn{6}{|l|}{ Age (years) } \\
\hline Mean $\pm S D$ & $44.6 \pm 13.2$ & $45.0 \pm 13.2$ & $45.4 \pm 12.4$ & $44.8 \pm 13.2$ & $0.096^{\mathrm{a}}$ \\
\hline Min; max & $19 ; 83$ & $21 ; 83$ & $24 ; 79$ & $19 ; 83$ & \\
\hline \multicolumn{6}{|l|}{ Gender } \\
\hline Male, $n(\%)$ & $684(45.9)$ & $182(45.9)$ & $34(50.1)$ & $900(46.1)$ & $0.866^{\mathrm{a}}$ \\
\hline Female, $n(\%)$ & $805(54.1)$ & $214(54.1)$ & $33(49.9)$ & $1052(53.9)$ & \\
\hline \multicolumn{6}{|c|}{ Goligher HD grade, $n(\%)$} \\
\hline I & $452(30.4)$ & $42(8.5)$ & 0 & $494(25.3)$ & \\
\hline II & $736(49.4)$ & $154(17.3)$ & 0 & $890(45.6)$ & \\
\hline III & $263(17.7)$ & $199(43.1)$ & 0 & $462(23.7)$ & \\
\hline IV & $38(2.6)$ & $1(0.9)$ & $67(63.2)$ & $106(5.4)$ & \\
\hline \multicolumn{6}{|c|}{ Patients with risk factors for HD, $n(\%)$} \\
\hline \multicolumn{6}{|l|}{ Constipation present } \\
\hline $\begin{array}{l}\text { Experienced } \\
\text { for } \leq 18 \text { months }\end{array}$ & $183(9.4)$ & & & & \\
\hline $\begin{array}{l}18 \text { months to } \\
5 \text { years }\end{array}$ & $152(7.8)$ & & & & \\
\hline$>5$ years & $220(11.3)$ & & & & \\
\hline $\begin{array}{l}\text { Bristol stool } \\
\text { chart type } 1-2 \\
\text { stools }\end{array}$ & $483(24.7)$ & & & & \\
\hline $\begin{array}{l}\text { Regular use of } \\
\text { laxatives }\end{array}$ & $223(11.4)$ & & & & \\
\hline
\end{tabular}

${ }^{a}$ Wilcoxon signed-rank test 
Table 2 Number (\%) of patients with clinical manifestations of hemorrhoids prior to initiation of conservative treatment

\begin{tabular}{lllll}
\hline Major complaints & \multicolumn{4}{l}{ Goligher hemorrhoid grade } \\
\cline { 2 - 5 } & Grade I $(\boldsymbol{n}=\mathbf{4 9 4})$ & Grade II $(\boldsymbol{n}=\mathbf{8 9 0})$ & Grade III $(\boldsymbol{n = 4 6 2})$ & Grade IV $(\boldsymbol{n}=\mathbf{1 0 6})$ \\
\hline Prolapse & $34(6.9)$ & $467(52.5)$ & $400(86.6)$ & $104(98.1)$ \\
Anal pain & $296(59.9)$ & $572(64.3)$ & $292(63.2)$ & $81(76.4)$ \\
Anal bleeding & $283(57.3)$ & $640(71.9)$ & $374(81.0)$ & $92(86.8)$ \\
Perianal edema & $263(53.2)$ & $529(59.4)$ & $259(56.1)$ & $69(65.1)$ \\
\hline
\end{tabular}

Values are presented as number of patients (\%)

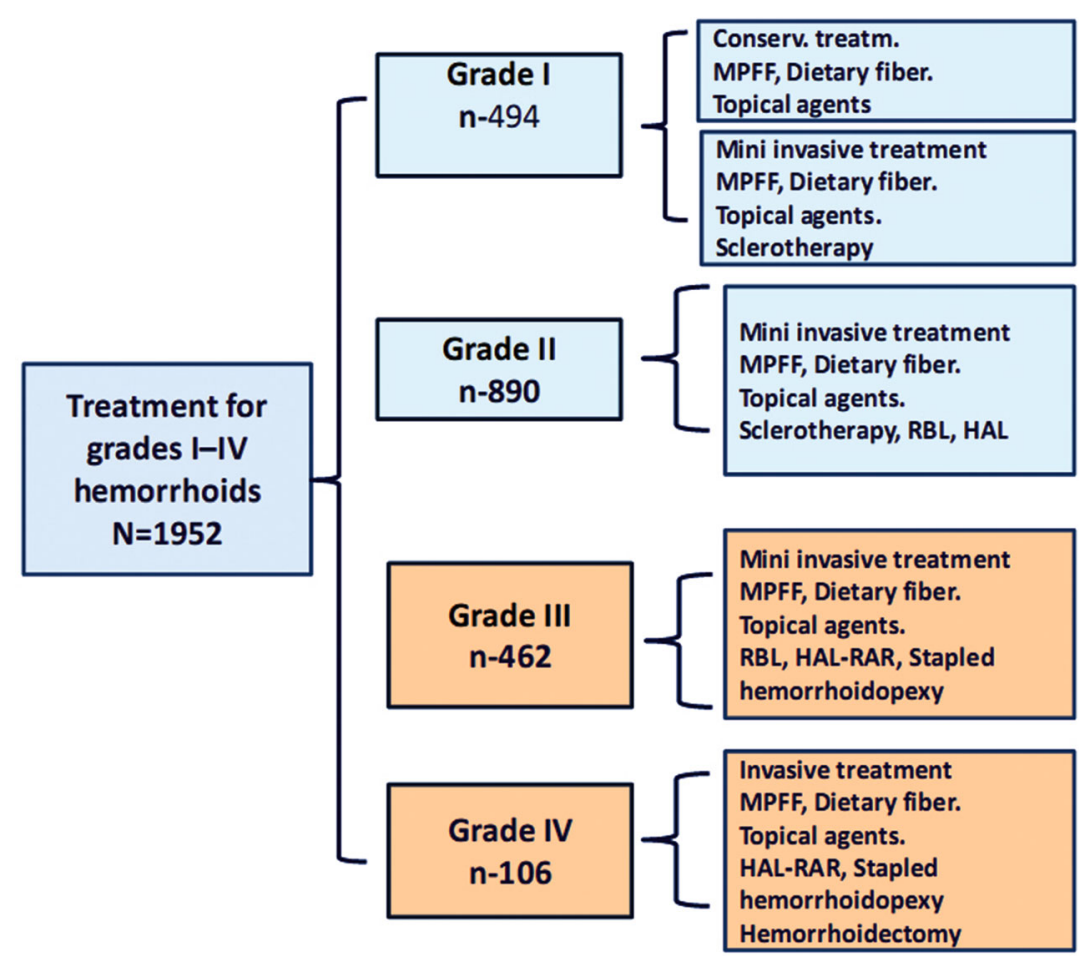

Fig. 1 Initial treatment for grades I-IV hemorrhoids (sclerotherapy, rubber band ligation $(\mathrm{RBL})$; minimally invasive treatment, Doppler-guided hemorrhoidal artery

symptoms) to 10 (the worst the symptoms have ever been). Stool consistency was described on the basis of the seven-category Bristol stool scale. Evaluation of the degree of prolapse of internal hemorrhoids was based on patients' responses and anoscopy, which is a standard practice for determining the degree of prolapse of internal hemorrhoids. The study protocol specified terms for minimally invasive or ligation (DG-HAL); and invasive treatment, stapled hemorrhoidopexy and hemorrhoidectomy)

surgical treatment in cases when conservative treatment had no effect.

\section{Safety Assessment}

Safety assessment for the MPFF-based conservative treatment was performed during the follow-up visits. At each visit, any adverse events occurring in a patient were recorded in the CRF. 


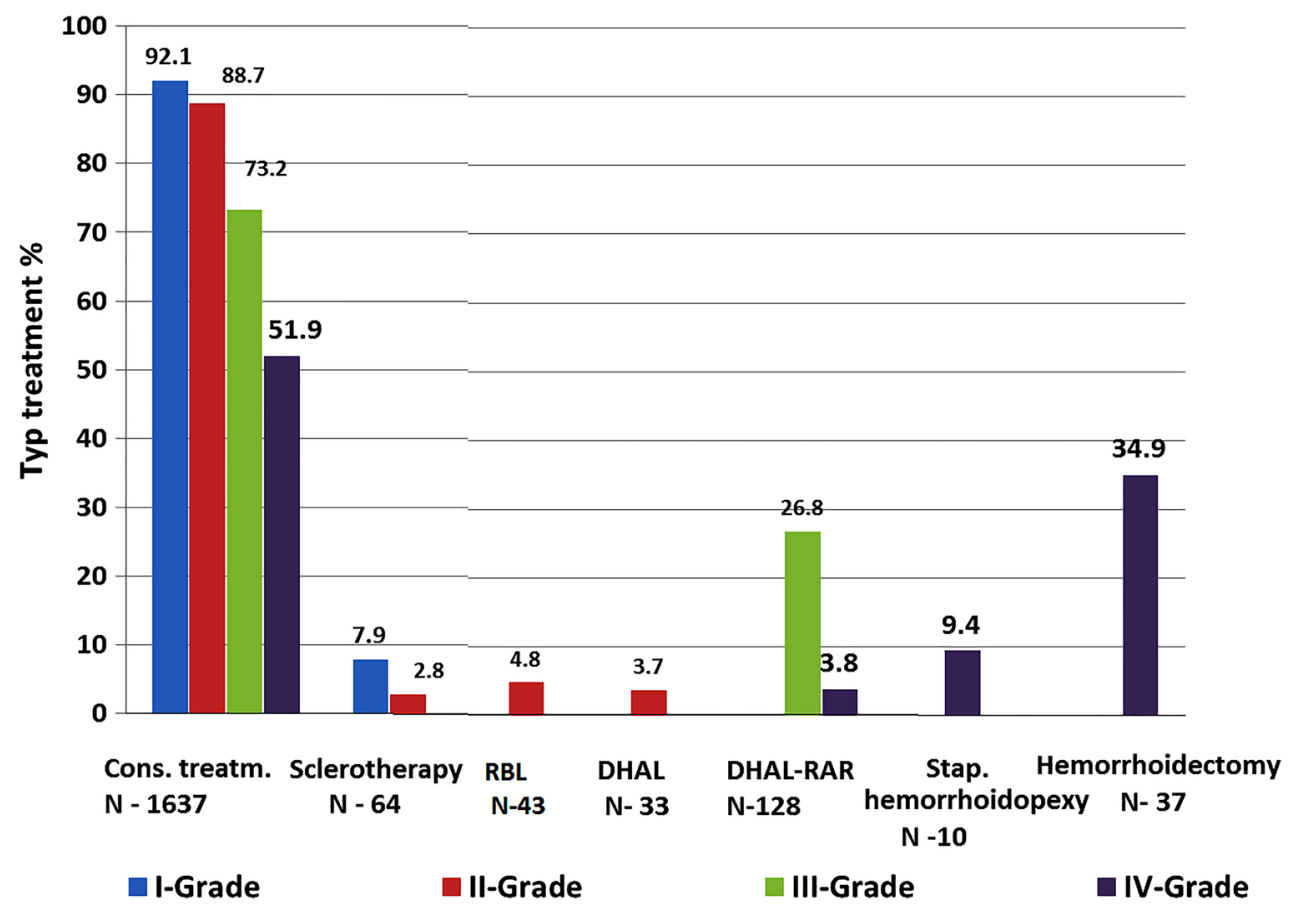

Fig. 2 Percentage of patients with conservative, minimally invasive, or invasive treatment depending on the degree of hemorrhoids

Table 3 Characteristics of treatment in the study group

\begin{tabular}{lc}
\hline Hemorrhoidal treatment & Patients, $\boldsymbol{n}$ (\%) \\
\hline MPFF & $1952(100.0)$ \\
Dietary fiber & $1201(60.9)$ \\
Topical agents & $1646(83.5)$ \\
NSAIDS & $397(20.1)$ \\
Minimally invasive treatment & $396(20.3)$ \\
Invasive treatment & $67(3.4)$ \\
\hline
\end{tabular}

All adverse events were evaluated by an investigator in relation to the presence or absence of a causal relationship with MPFF use and by degree of severity. Special situations such as suspected transmission of an infectious agent via a drug, overdose, abuse, misuse, off-label use, use during pregnancy or breastfeeding, medication error or occupational exposure, lack of efficacy, as well as adverse events related to the use of low-quality or falsified drug were also recorded.

\section{Statistical Analyses}

Statistical analyses were carried out using the SPSS software (version 18.0, Chicago, IL). Continuous data fitting the normal distribution are presented as mean and standard deviation, or median and range. For the comparisons of treatment results by nominal variables, Wilcoxon signed-rank test for matched samples or the Mann-Whitney $U$ test was used. Differences were considered statistically significant at $p<0.005$.

\section{RESULTS}

The study included 1952 patients with hemorrhoids who met the selection criteria. Mean age was $44.8 \pm 13.2$ years (range $19-83$ years). Hemorrhoid severity was assessed using the Goligher classification [11]. Conservative treatment was effective in 1489 out of 1952 (76.3\%) patients. Invasive treatment was performed in 68 (3.5\%) patients with grade IV HD. Conservative therapy with MPFF was combined with 


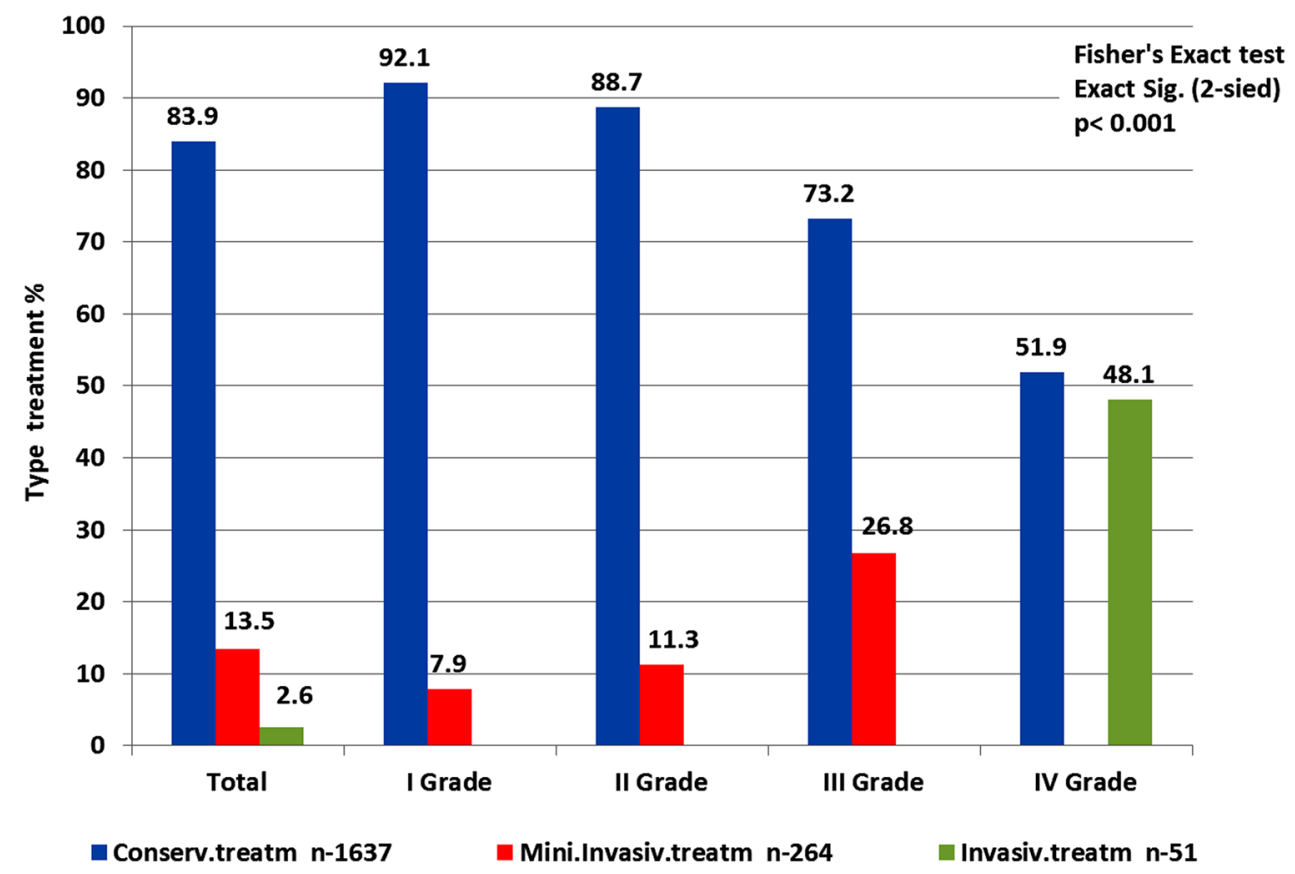

Fig. 3 Percentages of patients with successful conservative treatment or surgery according to the grade of hemorrhoidal disease

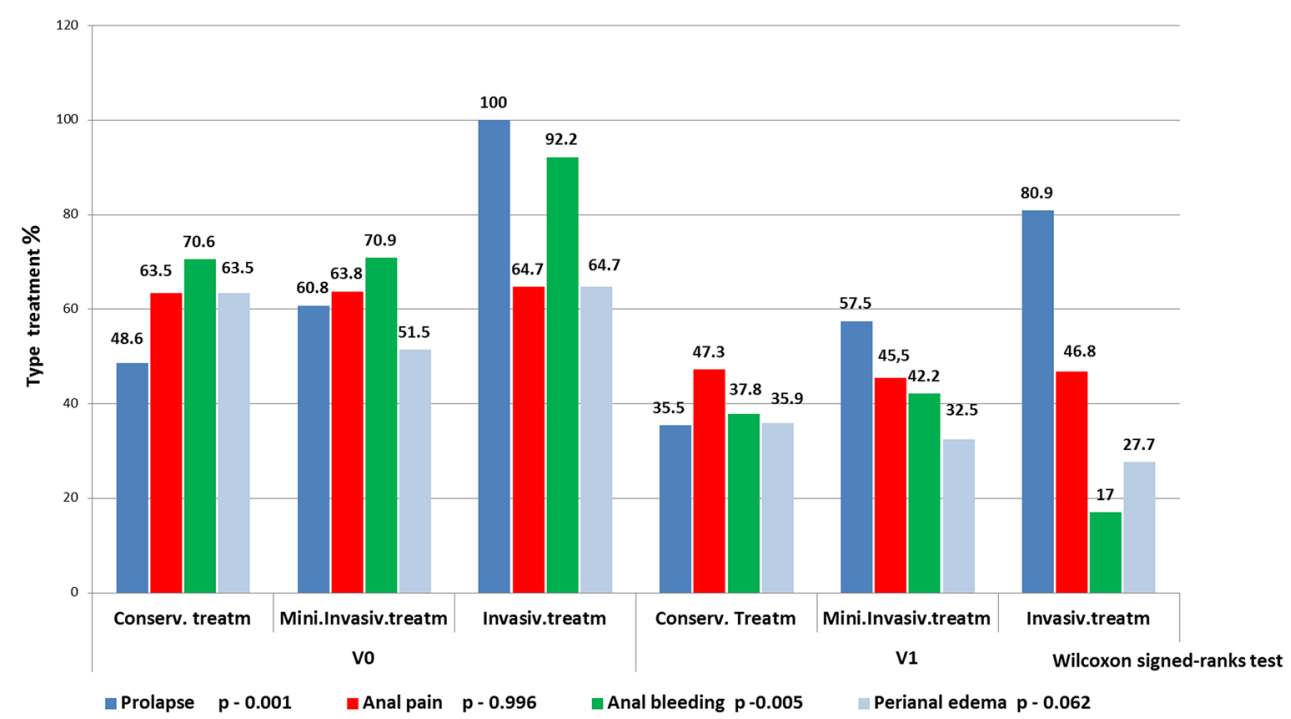

Fig. 4 Clinical outcomes after 5-7 days of conservative treatment

minimally invasive treatment in 395 (20.2\%) patients with grade I-III HD. The main characteristics of the study patients are presented in Table 1 and the clinical manifestations of HD prior to the initiation of conservative treatment are presented in Table 2 .
According to self-reported data, 555 (28.4\%) patients had constipation, 483 (24.7\%) had type 1-2 stools, 222 (11.4\%) had type 5-7 stools, and $873(44.7 \%)$ had a prolonged defecation time of from 6 to $30 \mathrm{~min}$. Analysis of clinical manifestations (V0) showed that all patients had an exacerbation of HD. Conservative therapy based 


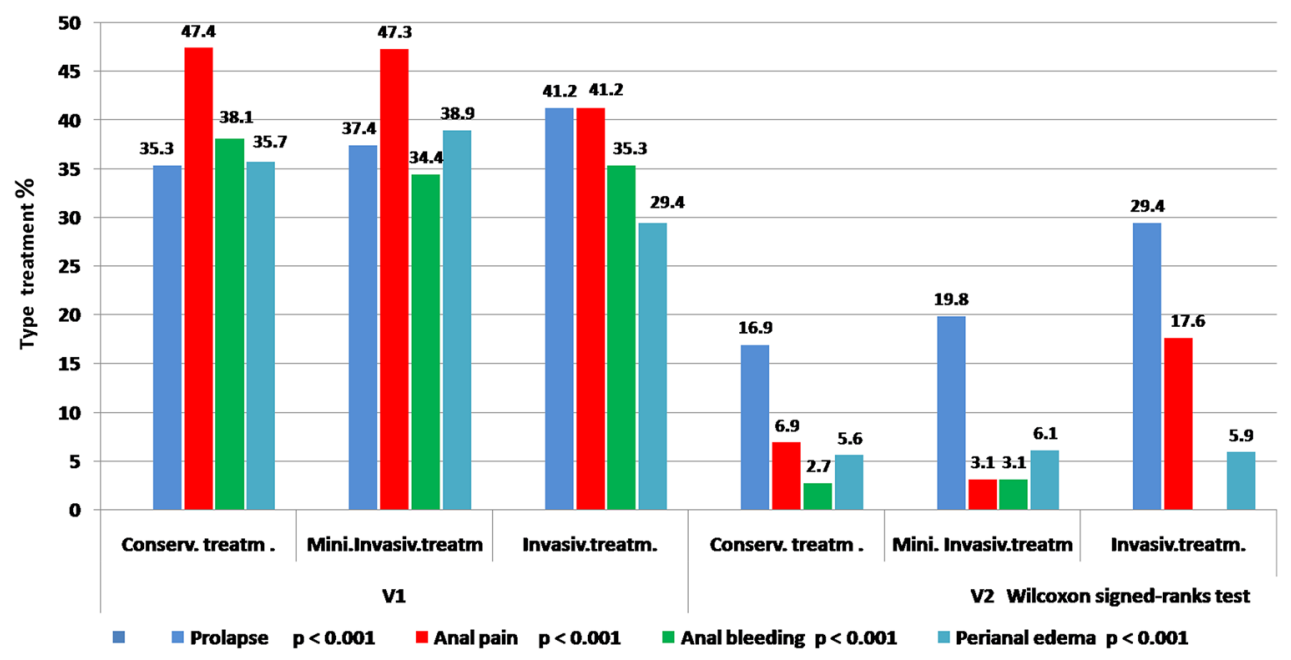

Fig. 5 Clinical outcomes after 25-30 days of conservative treatment

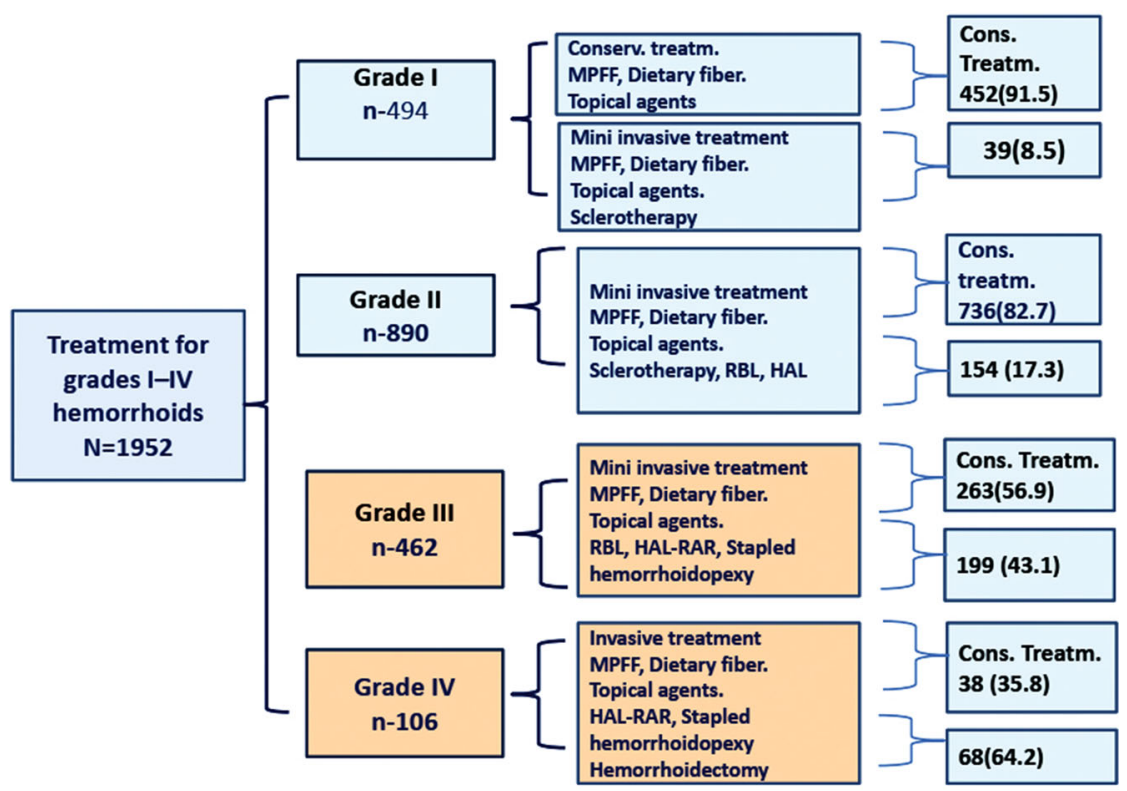

Fig. 6 Type and effectiveness of the treatment of I-IV degree hemorrhoids in the period V0-V2

on MPFF was started and, if ineffective, patients were recommended for minimally invasive or invasive surgical treatment (Figs. 1, 2).

All patients $(N=1952)$, regardless of invasive or conservative treatment, received phlebotonic treatment with MPFF at a dose of $1000 \mathrm{mg}$ /day. In 1916 cases $(98.2 \%)$, patients were treated with MPFF for more than 4 weeks. The concomitant use of local agents (suppositories, ointments) was reported in 1646 (83.5\%) patients, and intake of dietary fiber (medications based on P. ovata seed shells) was prescribed in $1201 \quad(60.9 \%)$ patients. The treatments patients received during the study are presented in Table 3 .

The type of treatment by HD severity is presented in Fig. 3 . The treatment results for the first 5-7 days (V0-V1) showed that MPFF-based conservative treatment was effective in 1637 $(83.9 \%)$ patients $(p<0.001)$ including most 


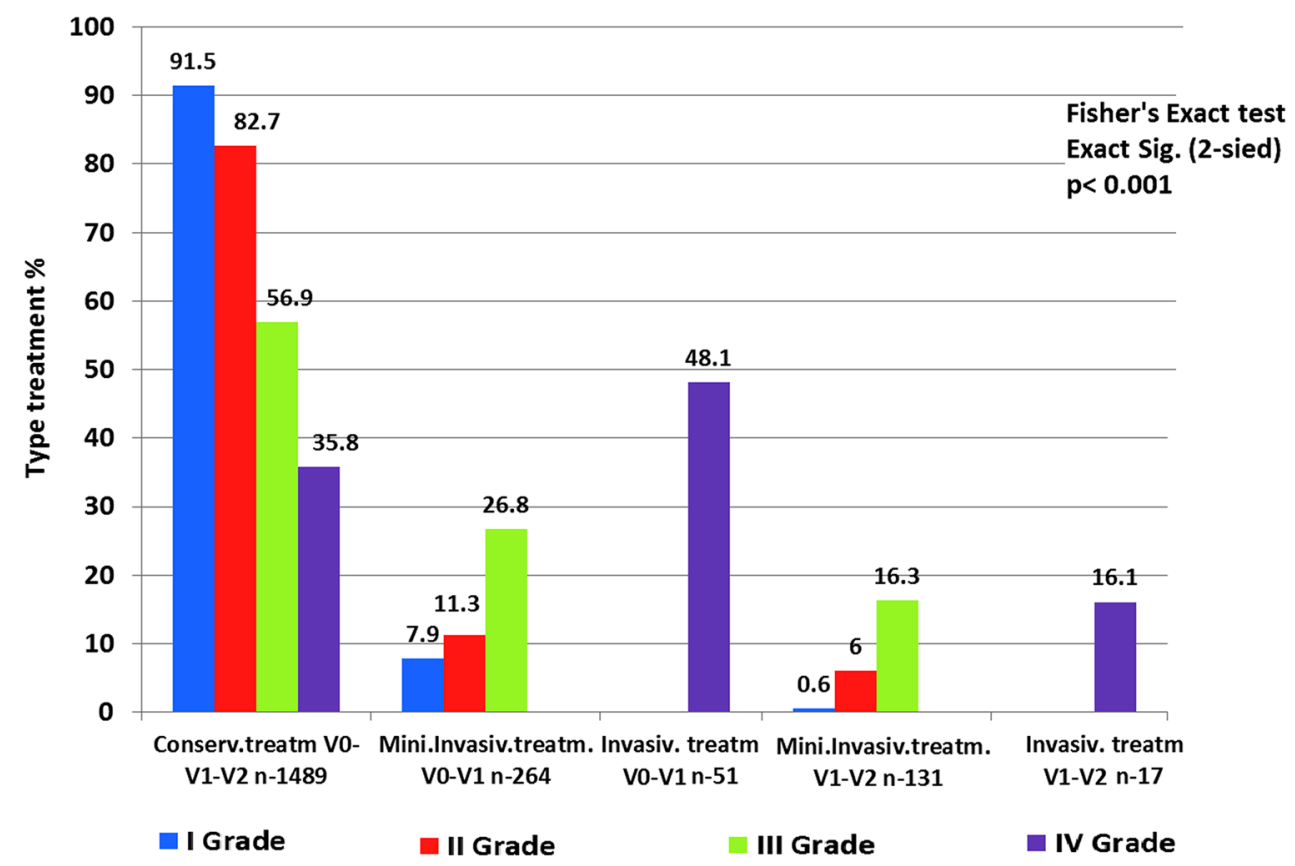

Fig. 7 Ratio of patients with successful conservative, minimally invasive, or invasive treatment according to the grade of hemorrhoidal disease, in the first 25-30 days (V1-V2)

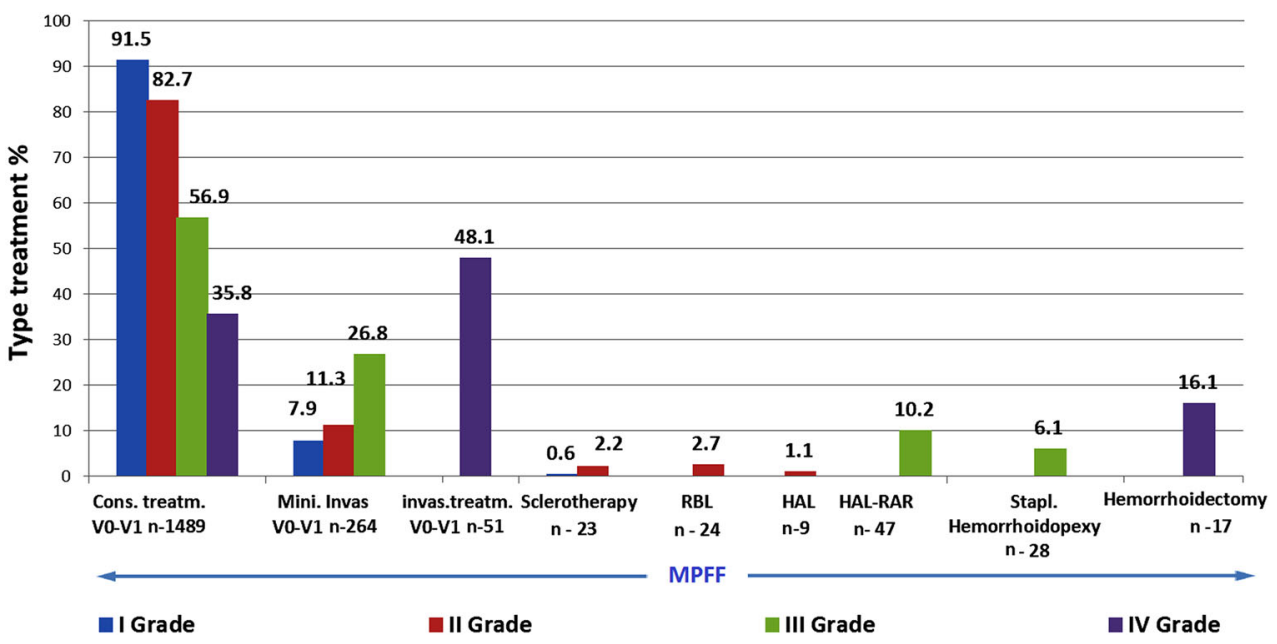

Fig. 8 Percentage of patients with conservative, minimally invasive, or invasive treatment depending on the degree of hemorrhoids (V1-V2)

patients with grade I-III HD and a proportion of patients with grade IV HD (Fig. 3).

There was a reduction in the main hemorrhoidal symptoms, including prolapse, bleeding, swelling, and pain (Fig. 4). The assessment using the VAS revealed a significant $(p<0.001)$ alleviation of pain from $4.69 \pm 0.95(0-10) \mathrm{cm}$ at baseline to $2.73 \pm 0.38 \quad(0-8) \quad \mathrm{cm}$ after 5-7 days. In 268 (13.7\%) patients, treatment of grade I-III HD was combined with minimally invasive treatment. Invasive treatment was performed in 47 (2.4\%) patients with grade IV HD in whom no regression of symptoms was noted. 
Table 4 Number (\%) of patients with relapse of clinical manifestations of hemorrhoids according to the grade of disease

\begin{tabular}{|c|c|c|c|c|c|}
\hline \multirow{2}{*}{$\begin{array}{l}\text { Rates of } \\
\text { relapse }\end{array}$} & \multirow{2}{*}{$\begin{array}{l}\text { All } \\
(n=1952)\end{array}$} & \multicolumn{4}{|c|}{ Goligher grade of hemorrhoids } \\
\hline & & $\begin{array}{l}\text { Grade I } \\
(n=494)\end{array}$ & $\begin{array}{l}\text { Grade II } \\
(n=890)\end{array}$ & $\begin{array}{l}\text { Grade III } \\
(n=462)\end{array}$ & $\begin{array}{l}\text { Grade IV } \\
(n=106)\end{array}$ \\
\hline No symptoms & $995(51.0)$ & $234(47.4)$ & $513(57.6)$ & $213(46.1)$ & $35(33.0)$ \\
\hline Rarely & $635(32.5)$ & $150(30.4)$ & $269(30.2)$ & $169(36.6)$ & $47(44.3)$ \\
\hline Sometimes & $253(13.0)$ & $79(16.0)$ & $89(10.0)$ & $69(14.9)$ & $16(15.1)$ \\
\hline Regularly & $64(3.3)$ & $29(5.9)$ & $17(1.9)$ & $10(2.2)$ & $8(7.5)$ \\
\hline All the time & $1(0.1)$ & - & $1(0.1)$ & - & - \\
\hline No data & $4(0.2)$ & $2(0.4)$ & $1(0.1)$ & $1(0.2)$ & - \\
\hline
\end{tabular}

During the second follow-up period (V1-V2), all patients, including patients who underwent minimally invasive or invasive treatment in the first period, continued to receive phlebotrophic therapy with MPFF. Analysis of the second period of treatment shows a significant reduction in the main symptoms of hemorrhoids in all study groups compared to the first period (V0-V1) $(p<0.001)$ (Fig. 5). The analysis of CRFs revealed that conservative therapy was effective in 1489 (76.3\%) patients. Conservative therapy with the use of MPFF was effective in patients with grade $1(n=452,91.5 \%)$ and grade II ( $n=736,82.7 \%)$ HD. The combination of conservative therapy and minimally invasive treatment was required in $42(8.5 \%)$ of patients with grade I HD and in 154 (17.3\%) of patients with grade II HD. Among patients with grades III and IV HD, 199 (43.1\%) and 68 (64.2\%), respectively, required minimally invasive or invasive treatment in addition to conservative therapy. The effectiveness of treatment for grade I-IV HD is presented in Fig. 6.

These data suggest that phlebotrophic therapy (for more than 3 weeks) reduces the degree of prolapse of internal hemorrhoids and bleeding episodes, but does not completely cure them (Fig. 6). At the V2 visit (25-30 days), pain was reported in only 125 (6.4\%) patients, and it was of mild severity [VAS score $1.70 \pm 0.13$ (1-7) $\mathrm{cm} ; p<0.001]$.

In some patients, conservative therapy was combined with minimally invasive or invasive treatment. Minimally invasive treatment was performed in $131(6.7 \%)$ patients with grade
I-III HD and 17 (6.1\%) patients with grade IV HD. The nature of the treatment in the period V1-V2 is shown in Figs. 7 and 8. At the end of conservative treatment with MPFF, 995 (51.0\%) patients with HD of any grade at baseline were free of symptoms (Table 4).

\section{Adverse Events During Treatment}

Treatment-emergent adverse events were reported in $2(0.10 \%)$ patients and consisted of skin rash, and swelling of the veins of the hands with a burning sensation and itching in the hands. According to the investigator's assessment, the events were not considered serious in either patient, but were related to intake of MPFF and resulted in drug withdrawal.

\section{DISCUSSION}

Hemorrhoidal disease is the most prevalent pathology of the anorectal region in adult populations worldwide $[1,12]$ and is associated with major medical and socioeconomic issues. Exacerbations have a significant effect on patients' quality of life and result in lost work days. Approximately $20 \%$ of patients will require surgical treatment and rehabilitation, which further increases recovery time and lost work days [12].

Current understanding of the pathogenesis of HD is based on two main pathologic factors. The first relates to the occurrence of pathologic arterial inflow through the branches of the 
superior rectal artery to the internal hemorrhoidal plexus and, as a consequence, the occurrence of an imbalance between arterial inflow and venous outflow from the cavernous tissue of the internal hemorrhoidal plexus, resulting in an abnormal dilation of the cavernous tissue [13]. Until recently, the mechanism of blood flow regulation in the hemorrhoidal plexus was not clear, as the increase in arterial inflow could not explain fully the development of prolapsed internal hemorrhoids.

A study by Aigner et al. [14] showed the presence of perivascular nerve fibers and smooth muscle sphincters regulating vascular diameter and, thus, the blood flow velocity. The decrease in the arterial lumen diameter leads to a temporary reduction in arterial inflow, which facilitates the venous outflow and cyclical drainage of the hemorrhoidal plexus. Failure of the autonomic regulation of submucosal anorectal vessels triggers a vicious circle with progressive dilation of blood vessels and impairment of venous outflow. As a result, the progressive hyperplasia of the cavernous tissue develops [14].

The second pathologic factor is related to the development of degenerative processes in the connective tissue of the hemorrhoidal plexus $[15,16]$. The progressive destruction of the ligament of Parks and the muscle of Treitz leads to the mobility of internal hemorrhoids and their shift in the anal canal. The physiologic relaxation of the internal anal sphincter allows venous outflow during a bowel movement through the expanding "hemorrhoidal cushions". The impairment of internal regulation of blood flow and the concomitant replacement of smooth muscle tissue by connective tissue are likely to be the key factors in the pathogenesis of $\operatorname{HD}[14,17,18]$.

It is widely believed that one of the risk factors for hemorrhoids is constipation and prolonged straining. The straining required to evacuate a solid fecal bolus may cause an increase in intra-abdominal pressure and lead to increased blood flow to the internal hemorrhoidal plexus and, consequently, to impaired venous outflow, which results in dilation of the hemorrhoidal plexus [15]. The evacuation of a solid fecal bolus is also thought to favor the shift of the hemorrhoidal cushion. However, recent studies have questioned the importance of constipation in the development of HD $[1,15,19]$. In particular, no significant relationship has been found between HD and constipation, and there have also been reports that diarrhea can act as a risk factor for HD development [5].

At present, general principles for both the prevention and conservative treatment of patients with HD include modification of dietary regimen and increase in fiber intake, which helps to avoid straining during defecation. Studies by Alonso-Coello et al. [20] have shown that the consumption of dietary fiber reduces clinical manifestations of HD by $50 \%$. At the same time, in patients with grade III and IV hemorrhoids who have prolapsed hemorrhoids, the intake of fiber is not effective [21].

The administration of venoactive agents was first described in the treatment of chronic venous insufficiency (CVI). In Russia, MPFF (Detralex) has been in use for more than 20 years. As a result of micronization of the drug to particles of less than $2 \mu \mathrm{m}$ and the synergism of action of the diosmin and hesperidin active components, drug absorption from the gastrointestinal tract is twice as fast as the regular form. In clinical practice, MPFF is therefore one of the most popular phlebotrophic drugs. It is also one of the most well-studied agents in this group with extensive data in the literature on the pharmacology of MPFF in clinical settings over the last three decades of clinical development and therapeutic use.

The pharmacological properties of MPFF have been demonstrated [22-36]:

- At the microcirculatory level with an effect on venous inflammatory processes leading to endothelial protection and a reduced inflammatory cascade from the early stages of venous inflammation to later stages involving skin changes

- On venous tone with a decrease in venous distensibility and an increase in elastic modulus and venous emptying with an optimal dose/effect ratio obtained at a $1000 \mathrm{mg}$ daily dose 
- On microcirculatory permeability by reinforcing capillary resistance, decreasing capillary permeability, and improving microlymphatic drainage

A meta-analysis of 14 randomized trials of flavonoid agents in patients with HD has shown beneficial effects on the clinical symptoms and signs of the disease, reducing the risk of bleeding by $67 \%$, pain by $65 \%$, itching by $35 \%$, and relapse of HD by $47 \%$ [37]. Some researchers have also reported that MPFF can reduce discomfort, pain, and rates of secondary bleeding after hemorrhoidectomy [8, 38-41].

Topical treatment with various drugs is also prescribed for HD, and the main goal of such therapy is to reduce HD symptoms rather than to cure the disease itself. Traditionally, topical treatment includes administration of suppositories and ointments, which contain various active ingredients, such as local anesthetics, corticosteroids, antibiotics, and antiinflammatories.

Despite the lack of sufficient data to confirm the efficacy of diet and lifestyle modifications in the treatment of HD, many physicians include these recommendations as part of the conservative treatment of $H D$ and as a preventive measure. The recommendations usually include an increase in dietary fiber and fluid intake, and regulating stools in order to avoid straining during prolonged defecation [17, 42]. However, recommendations for conservative treatment should be based on the current understanding of the pathogenesis of HD. It is, therefore, important to consider not the presence or absence of constipation or diarrhea in a patient, but whether these syndromes change the synchronous mechanism of defecation, physiologic relaxation of the anal sphincter, and venous drainage of the hemorrhoidal plexus, and, as such, impair the pattern of blood flow in the hemorrhoidal plexus [14].

In the observational part of our study, 705 (36.1\%) patients reported a change in stool type, and $873(44.7 \%)$ indicated a prolongation of defecation time of more than 6 min. Taking the above into account, conservative treatment should be targeted at recovering motor function of the colon and restoring blood flow in the hemorrhoidal plexus, preferably with the use of systemic phlebotropic therapy.

The aim of our study was not to compare the effectiveness of conservative treatment using phlebotonics and the effectiveness of various methods of invasive or minimally invasive treatment of HD. Our population-based study aimed to obtain information on the effect of phlebotropic therapy on the evolution of clinical manifestations in patients with different degrees of internal hemorrhoids. HD symptoms affected patients from grade I disease, providing considerable rationale for the prescription of phlebotropic drugs, such as MPFF, to address the underlying cause, and dietary therapy and topical treatments to relieve symptoms. Conservative treatment with MPFF was shown to be effective for all grades of HD in patients who underwent minimally invasive and invasive treatment and in those who did not. For the entire follow-up period, MPFF treatment was effective in 1489 (76.3\%) patients. In 463 $(23.7 \%)$ patients, treatment was combined with minimally invasive or invasive treatment: minimally invasive treatment was performed in 395 $(20.2 \%)$ patients with grade I-III HD, and invasive treatment in $68(3.5 \%)$ patients with grade IV HD.

Of particular interest is the finding that after the 30-day course of conservative treatment, $995(51.0 \%)$ patients were free of any clinical HD manifestations.

On the basis of the findings of the current study, conservative treatment of HD with the phlebotropic agent MPFF is effective for all grades of the disease. However, some patients with grade I-III HD will in addition require minimally invasive treatment, and some grade IV will require invasive treatment. At the same time, the combination of conservative treatment including MPFF with surgical treatment creates favorable conditions for a smooth postoperative period.

\section{CONCLUSION}

Studies on the pathophysiology of HD performed in the last decade have demonstrated an important role of dysregulation of vascular 
tone, leading to cavernous tissue hyperplasia, in the development of the disease. Conservative therapy with MPFF was effective in the relief of the main clinical manifestations of the disease, such as bleeding and prolapse of internal nodes, in the majority of patients at early stages of the disease, suggesting that the pathologic process may be reversible. The clinical effects of MPFF in patients with grade I and II HD were more conclusive than in those with grade III and IV hemorrhoids as a result of the development of irreversible degenerative changes in the ligamentous apparatus of the hemorrhoidal plexus. Nevertheless, in patients with more advanced HD, MPFF remains beneficial in preventing disease relapse and in promoting optimal conditions in the postoperative period.

\section{ACKNOWLEDGEMENTS}

The authors would like to thank Catherine Erina and Olga Linnik MD of Servier and Jenny Gricefor their support in medical writing coordination and management of this publication.

Funding. Servier was the financial and organizational partner for the CHORUS program, but did not have any influence on the analysis of the material, interpretation of the results and writing of the manuscript. Servier also funded medical writing assistance and the journal's article processing charges. All authors had full access to all of the data in this study, contributed to the concept and design of the study, were involved in the analysis and interpretation of the data, contributed to the drafting of the paper and approval of the final submitted version, and take complete responsibility for the integrity of the data and the accuracy of the data analysis.

Medical Writing Assistance. The authors would like to thank Catherine Yerina and Olga Linnik, MD, from Servier and Jenny Grice for their support in medical writing coordination and management of this publication. This support was funded by Servier.
Authorship. All named authors meet the International Committee of Medical Journal Editors (ICMJE) criteria for authorship for this article, take responsibility for the integrity of the work as a whole, and have given their approval for this version to be published.

Author Contributions. All authors contributed to the concept and design of the study, were involved in the analysis and interpretation of the data, and contributed to the drafting of the paper and approval of the final submitted version.

Disclosures. Evgeny A. Zagriadskiǐ has received consulting fees/remuneration for participation in advisory boards from Servier. Alexey M. Bogomazov has received consulting fees/remuneration for participation in advisory boards from Servier. Evgeny B. Golovko has received consulting fees/remuneration for participation in advisory boards from Servier.

Compliance with Ethics Guidelines. The study was conducted in accordance with the principles of the Declaration of Helsinki (version adopted in Fortaleza, Brazil, in 2013). All patients provided written informed consent to participate in the study. The study protocol (No. DIM-05682-003-RUS) was approved by local institutional ethics committees.

Open Access. This article is distributed under the terms of the Creative Commons Attribution-NonCommercial 4.0 International License (http://creativecommons.org/licenses/ by-nc/4.0/), which permits any noncommercial use, distribution, and reproduction in any medium, provided you give appropriate credit to the original author(s) and the source, provide a link to the Creative Commons license, and indicate if changes were made.

\section{REFERENCES}

1. Johanson JF, Sonnenberg A. The prevalence of hemorrhoids and chronic constipation: an epidemiologic study. Gastroenterology. 1990;98:380-6. 
2. Riss S, Weiser FA, Schwameis $\mathrm{K}$, et al. The prevalence of hemorrhoids in adults. Int J Colorectal Dis. 2012;27(2):215-20.

3. Madoff RD, Fleshman JW, Clinical Practice Committee, American Gastroenterological Association. American Gastroenterological Association technical review on the diagnosis and treatment of hemorrhoids. Gastroenterology. 2004;126:1463-73.

4. Medich DS, Fazio VW. Hemorrhoids, anal fissure, and carcinoma of the colon, rectum, and anus during pregnancy. Surg Clin N Am. 1995;75(1): 77-88.

5. Johanson JF, Sonnenberg A. Constipation is not a risk factor for hemorrhoids: a case-control study of potential etiological agents. Am J Gastroenterol. 1994;89:1981-6.

6. Kaidar-Person O, Person B, Wexner SD. Hemorrhoidal disease: a comprehensive review. J Am Coll Surg. 2007;204:102-17.

7. Acheson AG, Scholefield JH. Management of haemorrhoids. BMJ. 2008;336(7640):380-3.

8. Cospite M. Double-blind, placebo-controlled evaluation of clinical activity and safety of Daflon $500 \mathrm{mg}$ in the treatment of acute hemorrhoids. Angiology. 1994;45:566-73.

9. Perera N, Liolitsa D, Lype S, et al. Phlebotonics for haemorrhoids. Cochrane Database Syst Rev. 2012;8:CD004322.

10. World Medical Association. World Medical Association Declaration of Helsinki ethical principles for medical research involving human subjects. JAMA. 2013;310(20):2191-4.

11. Goligher JC. Haemorrhoids or piles. In: Golgher JC, editor. Surgery of the anus rectum and colon. 4th ed. London: Bailliere Tindall; 1980. p. 96.

12. Seok-Gyu S, Soung-Ho K. Optimal treatment of symptomatic hemorrhoids. J Korean Soc Coloproctol. 2011;27(6):277-81.

13. Aigner F, Bodner G, Gruber H, et al. The vascular nature of hemorrhoids. J Gastrointest Surg. 2006;10:1044-50.

14. Aigner F, Gruber H, Conrad F, et al. Revised morphology and hemodynamics of the anorectal vascular plexus: impact on the course of hemorrhoidal disease. Int J Colorectal Dis. 2009;24:105-13.

15. Loder PB, Kamm MA, Nicholls RJ, Phillips RK. Haemorrhoids: pathology, pathophysiology and aetiology. Br J Surg. 1994;81:946-54.
16. Morgado PJ, Suárez JA, Gómez LG, Morgado PJ. Histoclinical basis for a new classification of hemorrhoidal disease. Dis Colon Rectum. 1988;31:4744-80.

17. Lohsiriwat V. Hemorrhoids: from basic pathophysiology to clinical management. World J Gastroenterol. 2012;18:2009-17.

18. Lohsiriwat V. Approach to hemorrhoids. Curr Gastroenterol Rep. 2013;15:332.

19. Pigot F, Siproudhis L, Allaert FA. Risk factors associated with hemorrhoidal symptoms in specialized consultation. Gastroenterol Clin Biol. 2005;29:1270-4.

20. Alonso-Coello P, Mills E, Heels-Ansdell D, et al. Fiber for the treatment of hemorrhoids complications: a systematic review and metaanalysis. Am J Gastroenterol. 2006;101:181-8.

21. Moesgaard F, Nielsen ML, Hansen JB, Knudsen JT. High-fiber diet reduces bleeding and pain in patients with hemorrhoids: a double-blind trial of Vi-Siblin. Dis Colon Rectum. 1982;25:454-6.

22. Kastenis K. Micronized purified flavonoid fraction (MPFF): a review of its pharmacological effects, therapeutic efficacy and benefits in the management of chronic venous insufficiency. Curr Vasc Pharmacol. 2005;3(1):1-9.

23. Shoab SS, Porter J, Scurr JH, Coleridge-Smith PD. Endothelial activation response to oral micronised flavonoid therapy in patients with chronic venous disease-a prospective study. Eur J Vasc Endovasc Surg. 1999;17:313-8.

24. Boisseau MR. Leukocyte involvement in the signs and symptoms of chronic venous disease. Perspectives for therapy. Clin Hemorheol Microcirc. 2007;37:277-90.

25. Amiel M, Barbe R, Revel D. Plethysmographic study of the dose-effect relationship of Daflon $500 \mathrm{mg}$ in man. J Int Med. 1987;85(Suppl):16-8.

26. Amiel M, Barbe R. Study of the pharmacodynamic activity of Daflon $500 \mathrm{mg}$. Ann Cardiol Angiol. 1998;47(3):185-8.

27. Barbe R, Amiel M. Pharmacodynamic properties and therapeutic efficacy of Daflon ${ }^{\circledR} 500 \mathrm{mg}$. Phlebology. 1992;7(suppl 2):41-4.

28. Ibegbuna $\mathrm{V}$, Nicolaides $\mathrm{AN}$, Sowade $\mathrm{O}$, Leon $\mathrm{M}$, Geroulakos G. Venous elasticity after treatment with Daflon 500 mg. Angiology. 1997;48(1):45-9.

29. Allegra C, Bartolo MJR, Carioti B, Cassiani D. An original microhaemorheological approach to the 
pharmacological effects of Daflon $500 \mathrm{mg}$ in severe chronic venous insufficiency. Int J Microcirc Clin Exp. 1995;15(Suppl 1):50-4.

30. Allegra C, Bartolo MJR, Carioti B, Cassiani D, Besse Boffi MG. Microlymphography: assessment of Daflon $500 \mathrm{mg}$ activity in patients with chronic venous insufficiency. Phlebologie. 1999;52(3):353-6.

31. Behar A, Lagrue G, Cohen-Boulakia F, Baillet J. Study of capillary filtration by double labelling I131 albumin and Tc99 red cells. Application to the pharmacodynamic activity of Daflon ${ }^{\circledR} 500 \mathrm{mg}$. Int Angiol. 1988;2(7):35-8.

32. Belcaro G, Cesarone MR, De Sanctis MT, et al. Laser Doppler and transcutaneous oximetry: modern investigations to assess drug efficacy in chronic venous insufficiency. Int $\mathrm{J}$ Microcirc Clin Exp. 1995;15(Suppl. 1):45-9.

33. Galley P, Thiollet M. A double-blind, placebo-controlled trial of a new veno-active flavonoid fraction (S5682) in the treatment of symptomatic capillary fragility. Int Angiol. 1993;12:69-72.

34. Shoab SS, Porter J, Scurr JH, Coleridge-Smith PD. Effect of oral micronized purified flavonoid fraction treatment on leucocyte adhesion molecule expression in patients with chronic venous disease: a pilot study. J Vasc Surg. 2000;31:456-61.

35. Dimitroulopoulos D, Tsamakidis K, Xinopoulos D, Karaitianos I, Fotopoulou A, Paraskevas E. Prospective, randomized, controlled, observer-blinded trial of combined infrared photocoagulation and micronized purified flavonoid fraction versus each alone for the treatment of hemorrhoidal disease. Clin Ther. 2005;27:746-54.

36. Godeberge PH. Daflon $500 \mathrm{mg}$ is significantly more effective than placebo in the treatment of haemorrhoids. Phlebology. 1992;7(Suppl 2):61-3.

37. Alonso-Coello P, Zhou Q, Martinez-Zapata MJ, et al. Meta-analysis of flavonoids for the treatment of haemorrhoids. Br J Surg. 2006;93:909-20.

38. Misra MC, Parshad R. Randomized clinical trial of micronized flavonoids in the early control of bleeding from acute internal haemorrhoids. $\mathrm{Br} \mathrm{J}$ Surg. 2000;87(7):868-72.

39. Ho YH, Tan M, Seow-Choen F. Micronized purified flavonide fraction compared favorably with rubber band ligation and fiber alone in the management of bleeding hemorrhoids: randomized controlled trial. Dis Colon Rectum. 2000;43(1):66-9.

40. Ho YH, Foo CL, Seow-Choen F, Goh HS. Prospective randomized controlled trial of a micronized flavonide fraction to reduce bleeding after haemorrhoidectomy. Br J Surg. 1995;82(8):1034-5.

41. La Torre F, Nicolai AP. Clinical use of micronized purified flavonoid fraction for treatment of symptoms after hemorrhoidectomy: results of a randomized, controlled, clinical trial. Dis Colon Rectum. 2004;47:704-10.

42. Lohsiriwat V. Treatment of hemorrhoids: a coloproctologist's view. World J Gastroenterol. 2015;21(31):9245-52. 